\title{
NECESSARY AND SUFFICIENT CONDITIONS FOR GEOMETRIC MEANS OF SEQUENCES IN MULTIPLICATIVE CALCULUS
}

\author{
İ. ÇANAK, Ü. TOTUR, AND S. A. SEZER \\ Received 10 October, 2015
}

\begin{abstract}
In this paper we have introduced the concept of geometric convergence of a sequence and determined the necessary and sufficient condition under which convergence follows from geometric convergence of a sequence in multiplicative sense. Corollaries allow this condition to be replaced by multiplicative analogues of Schmidt type slow oscillation condition or Landau type two-sided condition.
\end{abstract}

2010 Mathematics Subject Classification: 26A06; 40A05; 40E05

Keywords: multiplicative calculus, geometric mean

\section{INTRODUCTION AND PRELIMINARIES}

During the 17th century many scholars, as Galileo, discussed the following problem:

"Two estimates, 10 and 1000, are proposed as the value of a horse, which estimates, if any, deviates more from the true value of 100?"

The scholars who maintained that deviations should be measured by differences concluded that the estimate of 10 was closer to the true value. However, Galileo eventually maintained that the deviations should be measured by ratios, and he concluded that two estimates deviated equally from the true value (see [8]).

In this situation, the question "If we measure by ratios, what kind of a calculus do we have?" appears. The answer is the main idea of multiplicative calculus. Multiplicative calculus is alternative to the classical calculus. It provides differentiation and integration tools based on multiplication instead of addition. Every fact in classical calculus has an analogue in multiplicative calculus.

In recent years, multiplicative calculus has attained noticeable importance and popularity, due mainly to its applications in different areas such as biomedical image analysis [7], physics [3], dynamical systems [2], numerical analysis [9], complex analysis [10], sequence spaces [5] and economy [6].

Now, we give some basic properties of multiplicative calculus. 
Let $x \in \mathbb{R}^{+}$. The multiplicative absolute value of $x$ (see [4]) is defined as

$$
|x|^{*}= \begin{cases}x, & \text { if } x \geq 1 \\ \frac{1}{x}, & \text { if } x<1 .\end{cases}
$$

Using the above definition we have the following properties of the multiplicative absolute value function.

For any $x, y \in \mathbb{R}^{+}$, the followings are valid (see [1]):

i) $|x|^{*} \geq 1$,

ii) $\left|\frac{1}{x}\right|^{*}=|x|^{*}$,

iii) $|x|^{*} \leq y$ if and only if $\frac{1}{y} \leq x \leq y$,

iv) $|x y|^{*} \leq|x|^{*}|y|^{*}$.

By means of the multiplicative absolute value, Bashirov et al. [4] introduced the multiplicative distance between positive real numbers $x$ and $y$ as

$$
d^{*}(x, y)=\left|\frac{x}{y}\right|^{*}
$$

which satisfies the following properties:

i) $d^{*}(x, y) \geq 1$ for all $x, y \in \mathbb{R}^{+}$,

ii) $d^{*}(x, y)=1$ if and only if $x=y$,

iii) $d^{*}(x, y)=d^{*}(y, x)$ for all $x, y \in \mathbb{R}^{+}$,

iv) $d^{*}(x, z) \leq d^{*}(x, y) d^{*}(y, z)$ for all $x, y, z \in \mathbb{R}^{+}$.

As a consequence, $\mathbb{R}^{+}$is a multiplicative metric space and a sequence $\left(x_{n}\right)$ in $\mathbb{R}^{+}$ converges to the limit $L \in \mathbb{R}^{+}$in the multiplicative sense if for all $\epsilon>1$, there exists $N \in \mathbb{N}$ such that $d^{*}\left(x_{n}, L\right)=\left|\frac{x_{n}}{L}\right|^{*}<\epsilon$ for all $n>N$, or equivalently,

$$
d^{*}\left(x_{n}, L\right) \stackrel{*}{\rightarrow} 1 \text { as } n \rightarrow \infty .
$$

It is well known that $\mathbb{R}^{+}$is not complete according to the Euclidean metric. To emphasize the importance of this work, we should first note that $\left(\mathbb{R}^{+},|.|^{*}\right)$ is a complete multiplicative metric space [1].

Also, it should be noted that throughout this paper the concept of convergence of a sequence is understood in multiplicative sense.

Let $\left(x_{n}\right)$ be a sequence of positive real numbers. The $n$-th geometric mean $g_{n}$ of $\left(x_{n}\right)$ is defined by

$$
g_{n}:=\left(\prod_{k=0}^{n} x_{k}\right)^{\frac{1}{n+1}}, \quad n=0,1,2, \ldots .
$$

We say that $\left(x_{n}\right)$ converges to $L$ by the geometric mean method, briefly: $G$-convergent to $L \in \mathbb{R}^{+}$if

$$
\lim _{n \rightarrow \infty} g_{n}=L
$$


and we write $x_{n} \stackrel{*}{\rightarrow} L(G)$.

If the sequence $\left(x_{n}\right)$ diverges, then we may assign a limit value to $\left(x_{n}\right)$ by a different limitation method. In this case we still say that $\left(x_{n}\right)$ diverges but, even so, we are able to find a finite number that can be considered as its limit value.

We can replace $\lim _{n \rightarrow \infty} x_{n}$, which possibly does not exist, by

$$
\lim _{n \rightarrow \infty}\left(\prod_{k=0}^{n} x_{k}\right)^{\frac{1}{n+1}}=L \in \mathbb{R}^{+}
$$

if this multiplicative limit exists and we use this value for the limit of the sequence $\left(x_{n}\right)$. This method is completely natural because it just takes geometric means and settles for computing a kind of "mean" limit where an actual limit fails to exist.

Our concern while studying a new kind of convergence is to determine whether it assigns the "correct" value to a sequence which is already convergent.

That is, does

$$
x_{n} \stackrel{*}{\rightarrow} L \quad \Longrightarrow \quad x_{n} \stackrel{*}{\rightarrow} L(G) ?
$$

Any method assigning a limit value to a sequence is said to be regular if this is the case. In Lemma 1, we prove that if the limit

$$
\lim _{n \rightarrow \infty} x_{n}=L
$$

exists, then the limit (1.1) also exists. That the converse of Lemma 1 is not true in general is provided by the following example.

Example 1. The sequence $\left(x_{n}\right)=\left(\prod_{k=0}^{n} e^{(-1)^{k}}\right)$ is divergent, but it is $G$-convergent to $\sqrt{e}$.

Here, we define the concept of slowly oscillation which is more general than the convergence of a sequence in multiplicative calculus.

Definition 1. A sequence $\left(x_{n}\right)$ of positive real numbers is said to be $*$-slowly oscillating if

$$
\liminf _{\lambda \rightarrow 1^{+}} \limsup _{n \rightarrow \infty} \max _{n<m \leq \lambda_{n}}\left|\frac{x_{m}}{x_{n}}\right|^{*}=1,
$$

where by $\lambda_{n}$ we denote the integral part of the product $\lambda n$, in symbol $\lambda_{n}:=[\lambda n]$, or equivalently, if for every $\epsilon>0$ there exists $n_{0}=n_{0}(\epsilon)$ and $\lambda_{0}=\lambda_{0}(\epsilon)>1$, as close to 1 as we wish, such that

$$
\left|\frac{x_{m}}{x_{n}}\right|^{*}<\epsilon
$$

whenever $n_{0}<n<m \leq \lambda_{n}$. 
It is also easy to show that (1.3) is satisfied if and only if $\lim \left|\frac{x_{m}}{x_{n}}\right|^{*}=1$ whenever $1<\frac{m}{n} \stackrel{*}{\rightarrow} 1, m, n \rightarrow \infty$.

Example 2. The sequence $\left(x_{n}\right)=(n)$ is $*$-slowly oscillating, but not convergent. In fact,

$$
\lim \left|\frac{x_{m}}{x_{n}}\right|^{*}=\lim \left|\frac{m}{n}\right|^{*}=1,
$$

whenever $1<\frac{m}{n} \stackrel{*}{\rightarrow} 1, m, n \rightarrow \infty$.

Our main goal in this paper is to obtain a necessary and sufficient condition under which convergence of a sequence follows from convergence of its geometric means, and vice versa.

\section{MAIN RESULT}

We prove the following theorem. In main theorem, some relations with convergence and $G$-convergence of a sequence are given. Also, we present some corollaries which include the multiplicative analogues of conditions of Schmidt type and Landau type.

Theorem 1. Let $\left(x_{n}\right)$ be a sequence of positive real numbers. If $\left(x_{n}\right)$ is $G$ convergent to a finite limit $L$, then $\left(x_{n}\right)$ is convergent to $L$ if and only if one of the following two conditions hold:

$$
\liminf _{\lambda \rightarrow 1^{+}} \limsup _{n \rightarrow \infty}\left(\left|\prod_{i=n+1}^{\lambda_{n}} \frac{x_{i}}{x_{n}}\right|^{*}\right)^{\frac{1}{\lambda_{n}-n}}=1
$$

or

$$
\liminf _{\lambda \rightarrow 1^{-}} \limsup _{n \rightarrow \infty}\left(\left|\prod_{i=\lambda_{n}+1}^{n} \frac{x_{n}}{x_{i}}\right|^{*}\right)^{\frac{1}{n-\lambda_{n}}}=1,
$$

where by $\lambda_{n}$ we denote the integral part of the product $\lambda n$, in symbol $\lambda_{n}:=[\lambda n]$.

The proof of Theorem 1 relies on the representations (3.4) and (3.5).

The next corollaries of Theorem 1 follows immediately.

Corollary 1. If the sequence $\left(x_{n}\right)$ is $G$-convergent to $L$ and $*$-slowly oscillating, then it is convergent to $L$.

Corollary 2. If $\left(x_{n}\right)$ is $G$-convergent to L and $\left(\left(\Delta^{*} x_{n}\right)^{n}\right)$ is bounded, where

$$
\Delta^{*} x_{n}=\frac{x_{n}}{x_{n-1}} \text { for } n \geq 1 \text { and } \Delta^{*} x_{0}=x_{0},
$$

then $\left(x_{n}\right)$ is convergent to $L$. 
Corollary 3. If $\left(x_{n}\right)$ is $G$-convergent to $L$ and

$$
\left(\Delta^{*} x_{n}\right)^{n} \stackrel{*}{\rightarrow} 1, \text { as } n \rightarrow \infty,
$$

then $\left(x_{n}\right)$ is convergent to $L$.

\section{AuXiLiary RESUlts}

We need following lemmas for the proof of our main theorem.

Lemma 1. If the sequence $\left(x_{n}\right)$ is convergent to $L$, then the sequence $\left(g_{n}\right)$ of its geometric means is convergent to $L$.

Proof. Let $\lim _{n \rightarrow \infty} x_{n}=L$. Then for all $\epsilon>1$, there exists $N_{0} \in \mathbb{N}$ such that $\left|\frac{x_{n}}{L}\right|^{*}<\epsilon^{1 / 2}$ whenever $n>N_{0}$, and $\left|\frac{x_{n}}{L}\right|^{*}<M$ whenever $n \leq N_{0}$. So, we have

$$
\begin{aligned}
\left|\frac{g_{n}}{L}\right|^{*} & =\left|\left(\prod_{k=0}^{n} x_{k}\right)^{\frac{1}{n+1}} /\left(\prod_{k=0}^{n} L\right)^{\frac{1}{n+1}}\right|^{*} \\
& =\left|\left(\prod_{k=0}^{n} \frac{x_{k}}{L}\right)^{\frac{1}{n+1}}\right|^{*} \\
& \leq\left(\prod_{k=0}^{n}\left|\frac{x_{k}}{L}\right|^{*}\right)^{\frac{1}{n+1}} \\
& =\left(\prod_{k=0}^{N_{0}}\left|\frac{x_{k}}{L}\right|^{*}\right)^{\frac{1}{n+1}}\left(\prod_{k=N_{0}+1}^{n}\left|\frac{x_{k}}{L}\right|^{*}\right)^{\frac{1}{n+1}} \\
& \leq M^{\frac{N_{0}+1}{n+1}}\left(\epsilon^{1 / 2}\right)^{\frac{n-N_{0}}{n+1}} \\
& \leq M^{\frac{N_{0}+1}{n+1}} \epsilon^{1 / 2}
\end{aligned}
$$

Since $\lim _{n \rightarrow \infty} M^{\frac{N_{0}+1}{n+1}}=1$, there exists $N_{1} \in \mathbb{N}$ such that $\left|M^{\frac{N_{0}+1}{n+1}}\right|^{*}<\epsilon^{1 / 2}$ whenever $n>N_{1}$. Therefore, there exists $N=\max \left\{N_{0}, N_{1}\right\}$ such that $\left|\frac{g_{n}}{L}\right|^{*}<\epsilon$ for all $n>N$. This completes the proof.

Next, we prove that if a sequence is $G$-convergent to a finite limit $L$, then the so-called moving geometric means converge to the same limit. More precisely, the following lemma is valid. 
Lemma 2. If the sequence $\left(x_{n}\right)$ is $G$-convergent to a finite limit $L$, then for each $\lambda>1$

$$
\tau_{n, \lambda_{n}}^{>}(x):=\left(\prod_{m=n+1}^{\lambda_{n}} x_{m}\right)^{\frac{1}{\lambda_{n}-n}} \stackrel{*}{\rightarrow} L,
$$

and for each $0<\lambda<1$

$$
\tau_{n, \lambda_{n}}^{<}(x):=\left(\prod_{m=\lambda_{n}+1}^{n} x_{m}\right)^{\frac{1}{n-\lambda_{n}}} \stackrel{*}{\rightarrow} L,
$$

where by $\lambda_{n}$ we denote the integral part of the product $\lambda n$, in symbol $\lambda_{n}:=[\lambda n]$.

Proof. If $\lambda>1$ and $n$ is large enough in the sense that $\lambda_{n}>n$, then

$$
\begin{aligned}
\left(\prod_{m=n+1}^{\lambda_{n}} x_{m}\right)^{\frac{1}{\lambda_{n}-n}} & =\left(\prod_{m=0}^{\lambda_{n}} x_{m} / \prod_{m=0}^{n} x_{m}\right)^{\frac{1}{\lambda_{n}-n}} \\
& =\left[\left(\left(\prod_{m=0}^{\lambda_{n}} x_{m}\right)^{\frac{1}{\lambda_{n}+1}}\right)^{\frac{\lambda_{n}+1}{\lambda_{n}-n}} /\left(\left(\prod_{m=0}^{n} x_{m}\right)^{\frac{1}{n+1}}\right)^{\frac{n+1}{\lambda_{n}-n}}\right] \\
& =\left(\left(g_{\lambda_{n}}\right)^{\frac{\lambda_{n}+1}{\lambda_{n}-n}} /\left(g_{n}\right)^{\frac{n+1}{\lambda_{n}-n}}\right) \\
& =g_{n}\left(\frac{g_{\lambda_{n}}}{g_{n}}\right)^{\frac{\lambda_{n}+1}{\lambda_{n}-n}}
\end{aligned}
$$

Since

$$
\left|\frac{\tau_{n, \lambda_{n}}^{>}(x)}{L}\right|^{*}=\left|\frac{g_{n}}{L}\right|^{*}\left(\left|\frac{g_{\lambda_{n}}}{L}\right|^{*}\right)^{\frac{\lambda n+1}{\lambda n-n}}\left(\left|\frac{g_{n}}{L}\right|^{*}\right)^{\frac{\lambda n+1}{\lambda n-n}},
$$

(3.1) follows from (1.1). The proof of (3.2) can be similarly obtained.

The following two representations of the ratio $\frac{x_{n}}{g_{n}}$ will be used in the proof of the main theorem.

Lemma 3. (i) For all $\lambda>1$ and large enough $n$, that is, when $\lambda_{n}>n$,

$$
\frac{x_{n}}{g_{n}}=\left(\frac{g_{\lambda_{n}}}{g_{n}}\right)^{\frac{\lambda_{n}+1}{\lambda_{n}-n}}\left[\left(\prod_{i=n+1}^{\lambda_{n}} \frac{x_{i}}{x_{n}}\right)^{\frac{1}{\lambda_{n}-n}}\right]^{-1} .
$$


(ii) For all $0<\lambda<1$ and large enough $n$, that is, when $n>\lambda_{n}$,

$$
\frac{x_{n}}{g_{n}}=\left(\frac{g_{n}}{g_{\lambda_{n}}}\right)^{\frac{\lambda_{n}+1}{n-\lambda_{n}}}\left[\left(\prod_{i=\lambda_{n}+1}^{n} \frac{x_{n}}{x_{i}}\right)^{\frac{1}{n-\lambda_{n}}}\right]^{-1} \text {. }
$$

Proof. By definition, for every $\lambda>1$, we have

$$
\begin{aligned}
\frac{g_{\lambda_{n}}}{g_{n}} & =\frac{\left(\prod_{i=0}^{\lambda_{n}} x_{i}\right)^{\frac{1}{\lambda_{n}+1}}}{\left(\prod_{i=0}^{n} x_{i}\right)^{\frac{1}{n+1}}} \\
& =\left(\prod_{i=0}^{n} x_{i}\right)^{\frac{n-\lambda_{n}}{(n+1)\left(\lambda_{n}+1\right)}}\left(\prod_{i=n+1}^{\lambda_{n}} x_{i}\right)^{\frac{1}{\lambda_{n}+1}}
\end{aligned}
$$

Hence,

$$
\begin{aligned}
\frac{g_{\lambda_{n}}}{g_{n}} & =g_{n} n^{\frac{n-\lambda_{n}}{\lambda n+1}}\left(\prod_{i=n+1}^{\lambda_{n}} \frac{x_{i}}{x_{n}} x_{n}\right)^{\frac{1}{\lambda_{n}+1}} \\
& =g_{n}{ }^{\frac{n-\lambda_{n}}{\lambda_{n}+1}}\left(\prod_{i=n+1}^{\lambda_{n}} \frac{x_{i}}{x_{n}}\right)^{\frac{1}{\lambda_{n}+1}} x_{n}^{\frac{\lambda_{n}-n}{\lambda_{n}+1}}
\end{aligned}
$$

and

$$
\left(\frac{g_{\lambda_{n}}}{g_{n}}\right)^{\frac{\lambda_{n}+1}{\lambda_{n}-n}}=\frac{x_{n}}{g_{n}}\left(\prod_{i=n+1}^{\lambda_{n}} \frac{x_{i}}{x_{n}}\right)^{\frac{1}{\lambda_{n}-n}}
$$

which is equivalent to (3.4).

(ii) The proof of (3.5) is similar.

\section{Proof}

Proof of Theorem 1. Necessity. Assume that (1.1) holds. Since $\left(x_{n}\right)$ converges to $L$, we have

$$
\lim _{n \rightarrow \infty}\left|\frac{x_{n}}{g_{n}}\right|^{*}=1 .
$$

It follows by (1.1) that for all $\lambda>1$,

$$
\lim _{n \rightarrow \infty}\left(\left|\frac{g_{\lambda_{n}}}{g_{n}}\right|^{*}\right)^{\frac{\lambda_{n}+1}{\lambda_{n}-n}}=1 .
$$


Since

$$
\left(\left|\prod_{i=n+1}^{\lambda_{n}} \frac{x_{i}}{x_{n}}\right|^{*}\right)^{\frac{1}{\lambda_{n}-n}} \leq\left|\frac{x_{n}}{g_{n}}\right|^{*}\left(\left|\frac{g_{\lambda_{n}}}{g_{n}}\right|^{*}\right)^{\frac{\lambda_{n}+1}{\lambda_{n}-n}}
$$

(2.1) follows from (3.4), (4.1), (4.2), and (4.3).

It follows by (1.1) that for all $0<\lambda<1$,

$$
\lim _{n \rightarrow \infty}\left(\left|\frac{g_{n}}{g_{\lambda_{n}}}\right|^{*}\right)^{\frac{\lambda_{n}+1}{n-\lambda_{n}}}=1
$$

Since

$$
\left(\left|\prod_{i=\lambda_{n}+1}^{n} \frac{x_{n}}{x_{i}}\right|^{*}\right)^{\frac{1}{n-\lambda_{n}}} \leq\left|\frac{x_{n}}{g_{n}}\right|^{*}\left(\left|\frac{g_{n}}{g_{\lambda_{n}}}\right|^{*}\right)^{\frac{\lambda_{n}+1}{n-\lambda_{n}}}
$$

(2.2) follows from (3.5), (4.1), (4.4), and (4.5).

Sufficiency. Assume the fulfilment of (1.1) and (2.1). It follows from (2.1) that there exists a decreasing sequence $\left(\lambda_{j}\right)$ converging to 1 such that

$$
\lim _{j \rightarrow \infty} \limsup _{n \rightarrow \infty}\left(\left|\prod_{i=n+1}^{\lambda_{j n}} \frac{x_{i}}{x_{n}}\right|^{*}\right)^{\frac{1}{\lambda_{j n}-n}}=1
$$

where $\lambda_{j n}=\left[\lambda_{j} n\right]$.

By (3.4), we have

$$
\limsup _{n \rightarrow \infty}\left|\frac{x_{n}}{g_{n}}\right|^{*} \leq \lim _{j \rightarrow \infty} \limsup _{n \rightarrow \infty}\left(\left|\frac{g_{\lambda_{j n}}}{g_{n}}\right|^{*}\right)^{\frac{\lambda_{j n}+1}{\lambda_{j n}-n}} \lim _{j \rightarrow \infty} \limsup _{n \rightarrow \infty}\left(\left|\prod_{i=n+1}^{\lambda_{j n}} \frac{x_{i}}{x_{n}}\right|^{*}\right)^{\frac{1}{\lambda_{j n}-n}}
$$

Taking (1.1) and (4.6) into account, we obtain

$$
\lim _{n \rightarrow \infty}\left|\frac{x_{n}}{g_{n}}\right|^{*}=1
$$

Assume the fulfilment of (1.1) and (2.2). It follows from (2.2) that there exists an increasing sequence $\left(\lambda_{j}\right)$ converging to 1 such that

$$
\lim _{j \rightarrow \infty} \limsup _{n \rightarrow \infty}\left(\left|\prod_{i=\lambda_{j n}+1}^{n} \frac{x_{n}}{x_{i}}\right|^{*}\right)^{\frac{1}{n-\lambda_{j n}}}=1
$$

where $\lambda_{j n}=\left[\lambda_{j} n\right]$. 
By (3.5), we have

$$
\limsup _{n \rightarrow \infty}\left|\frac{x_{n}}{g_{n}}\right|^{*} \leq \lim _{j \rightarrow \infty} \limsup _{n \rightarrow \infty}\left(\left|\frac{g_{n}}{g_{\lambda_{j n}}}\right|^{*}\right)^{\frac{\lambda_{j n}+1}{n-\lambda_{j n}}} \lim _{j \rightarrow \infty} \limsup _{n \rightarrow \infty}\left(\left|\prod_{i=\lambda_{j n}+1}^{n} \frac{x_{n}}{x_{i}}\right|^{*}\right)^{\frac{1}{n-\lambda_{j n}}}
$$

Taking (1.1) and (4.9) into account, we obtain

$$
\lim _{n \rightarrow \infty}\left|\frac{x_{n}}{g_{n}}\right|^{*}=1
$$

Combining (1.1) and (4.8) or (4.11), in either case we conclude that $\left(x_{n}\right)$ converges to $L$.

Since $*$-slow oscillation implies both (2.1) and (2.2), the proof of Corollary 1 follows from Theorem 1 . However, the proof of Corollary 1 seems to be fundamental, we prove it.

\section{ACKNOWLEDGEMENT}

The authors would like to thank the referee for very helpful suggestions and useful comments which improved the manuscript.

\section{REFERENCES}

[1] M. Abbas, B. Ali, and Y. I. Suleiman, "Common fixed points of locally contractive mappings in multiplicative metric spaces with application." Int. J. Math. Math. Sci., vol. 2015, no. Article ID 218683, p. 7 pages, 2015, doi: 10.1155/2015/218683.

[2] D. Aniszewska and M. Rybaczuk, "Analysis of the multiplicative Lorenz system." Chaos Solitons Fractals, vol. 25, no. 1, pp. 79-90, 2005, doi: 10.1016/j.chaos.2004.09.060.

[3] D. Aniszewska and M. Rybaczuk, "Chaos in Multiplicative Systems," in CHAOTIC SYSTEMS: THEORY AND APPLICATIONS, Skiadas, CH and Dimotikalis, I, Ed., doi: 10.1142/9789814299725_0002. PO BOX 128 FARRER RD, SINGAPORE 9128, SINGAPORE: WORLD SCIENTIFIC PUBL CO PTE LTD, 2010, Proceedings Paper, pp. 9-16, 2nd Chaotic Modeling and Simulation International Conference (CHAOS2009), Chania, GREECE, JUN 0105, 2009.

[4] A. E. Bashirov, E. M. Kurpınar, and A. Özyapıcı, "Multiplicative calculus and its applications." J. Math. Anal. Appl., vol. 337, no. 1, pp. 36-48, 2008, doi: 10.1016/j.jmaa.2007.03.081.

[5] A. F. Çakmak and F. Başar, "Some new results on sequence spaces with respect to non-Newtonian calculus.” J. Inequal. Appl., vol. 2012, p. 17, 2012, doi: 10.1186/1029-242X-2012-228.

[6] D. A. Filip and C. Piatecki, "A non-Newtonian examination of the theory of exogenous economic growth." Math. Aeterna, vol. 4, no. 2, pp. 101-117, 2014.

[7] L. Florack and H. Van Assen, "Multiplicative calculus in biomedical image analysis." J. Math. Imaging Vis., vol. 42, no. 1, pp. 64-75, 2012, doi: 10.1007/s10851-011-0275-1.

[8] M. Grossman and R. Katz, "Non-Newtonian calculus.” Pigeon Cove, Mass.: Lee Press. VIII, 94 p. $\$ 6.00$ (1972)., 1972.

[9] E. Misirli and Y. Gurefe, "Multiplicative Adams Bashforth-Moulton methods." Numer. Algorithms, vol. 57, no. 4, pp. 425-439, 2011, doi: 10.1007/s11075-010-9437-2. 
[10] A. Uzer, "Multiplicative type complex calculus as an alternative to the classical calculus." Comput. Math. Appl., vol. 60, no. 10, pp. 2725-2737, 2010, doi: 10.1016/j.camwa.2010.08.089.

Authors' addresses

I. Çanak

Ege University, Department of Mathematics, 35100 Izmir, Turkey

E-mail address: ibrahim.canak@ege.edu.tr

Ü. Totur

Adnan Menderes University, Department of Mathematics, 09010 Aydin, Turkey

E-mail address: utotureadu.edu.tr

\section{S. A. Sezer}

Istanbul Medeniyet University, Department of Mathematics, 35100 Izmir, Turkey

E-mail address: sefaanil. sezer@medeniyet.edu.tr 